\title{
Emerging pathogens: new identifications from Australasia and contribution of electron microscopy to the detection of Batrachochytrium dendrobatidis and management of chytridiomycosis.
}

Alex D. Hyatt, Sandra Crameri, Elishia Mackay, Diane Green, Ruben-Lee Roennfeldt ${ }^{1}$ and the Cytridiomycosis Group*

Australian Animal Health Laboratory (AAHL), CSIRO Livestock Industries, Private Bag 24, Geelong, Victoria 3220 Australia. Email: alex.hyatt@,csiro.au.

${ }^{1}$ School of Life and Environmental Sciences Deakin University PO Box 423 Warrnambool, Victoria, Australia 3280.

* Lee Berger, Rick Speare, Peter Daszak, Andrew Cunningham, Donna Boyle, David Boyle, Alan Marshall and Veronica Olsen.

New pathogens continue to be identified. Within the past 18 months new viruses have been identified in Australasia. The agents have been identified from cell cultures and/or tissues arising from a range of animals including abalone, amphibians, copepods and bats. Identification of each agent is undertaken in a methodical way whereby discrete datasets are generated via collection of information via negative contrast electron microscopy (NCEM), examination of ultrathin sections and where possible immuno-electron microscopy. The datasets are then used as demarcation criteria to identify the agents to the level of family, or if possible, genus. Of interest is that many of these infectious agents may arguably be inconsequential whilst others have profound impacts on biodiversity, commerce/trade and even human health.

Not all new diseases are caused by viruses. Chytridiomycosis for example is a deadly disease of amphibians and is caused by a highly infectious fungus Batrachochytrium dendrobatidis $(B d)$. The fungus belongs to the phylum Chytridiomycota and order Chytridiales. The disease is now recognised as a fatal disease of amphibians and is the most common disease of frogs worldwide. It infects two amphibian orders (Anura and Caudata), 14 families and at least 200 species and is responsible for at least one species extinction. Chytridiomycosis is now a notifiable disease by the OIE (International des Epizooties - World Organisation for Animal Health).

So what made chytridiomycosis such a significant disease? How did it proliferate throughout the world, what makes it so deadly and how can its spread and impact of disease be mitigated? To address these questions a list of important objectives were identified by an international panel of herpetologists. The top priorities were to identify the aetiological agent and to develop diagnostic assays and sampling protocols. Only through the development and validation of these could other studies including surveys, understanding pathogenesis and effective management programs be effectively undertaken.

Electron microscopy was the diagnostic tool responsible for the identification of $B d$. Following identification, transmission and scanning electron microscopy were used to understand the biology of the disease from which diagnostic assays and associated sampling techniques were developed. 
The assays included a range of histochemical protocols, immuno-electron microscopy and one molecular technique (TaqMan real time PCR). The techniques were validated and their respective sensitivities and specificities (analytical and diagnostic) determined. This work has now underpinned other important studies (refer above) intended to reduce the impact of the disease and thereby facilitate the conservation of many threatened and endangered species.

This presentation will describe the search for the aetiological agent and development of assays from a microscopical perspective. It will highlight the use of conventional, immuno- and cryotransmission and scanning electron microscopy in unraveling the identification of the fungus and the development of internationally validated and accepted diagnostic assays. The ongoing requirement for international collaboration in respect to identify new infectious agents will be highlighted together with the need for rapid turn-around times.

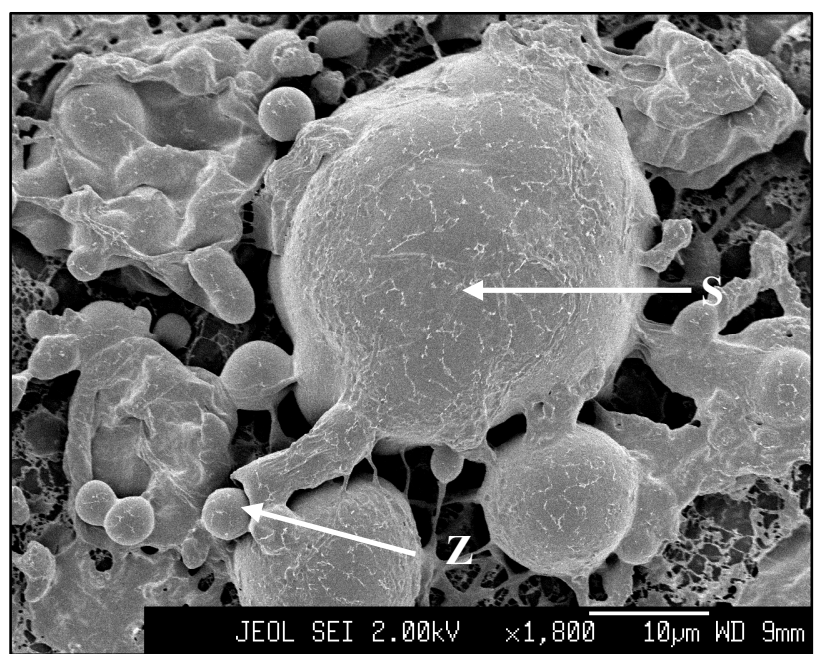

Figure 1. Scanning electron micrograph of a frozen hydrated culture of $B d$. $\mathrm{S}$, zoosporangium; $\mathrm{Z}$, zoospore.

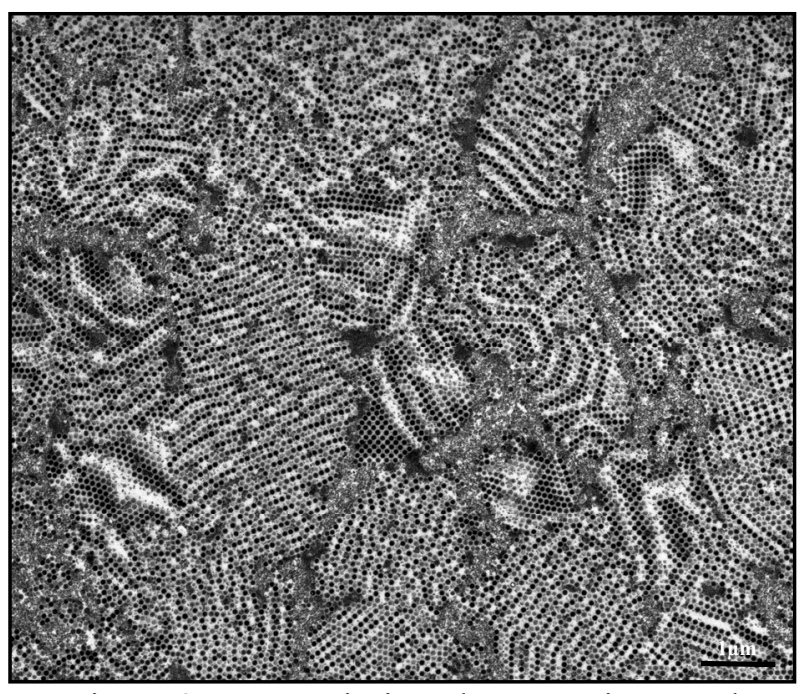

Figure 3. Transmission electron micrograph of an ultrathin section from an invertebrate (Crustacea).

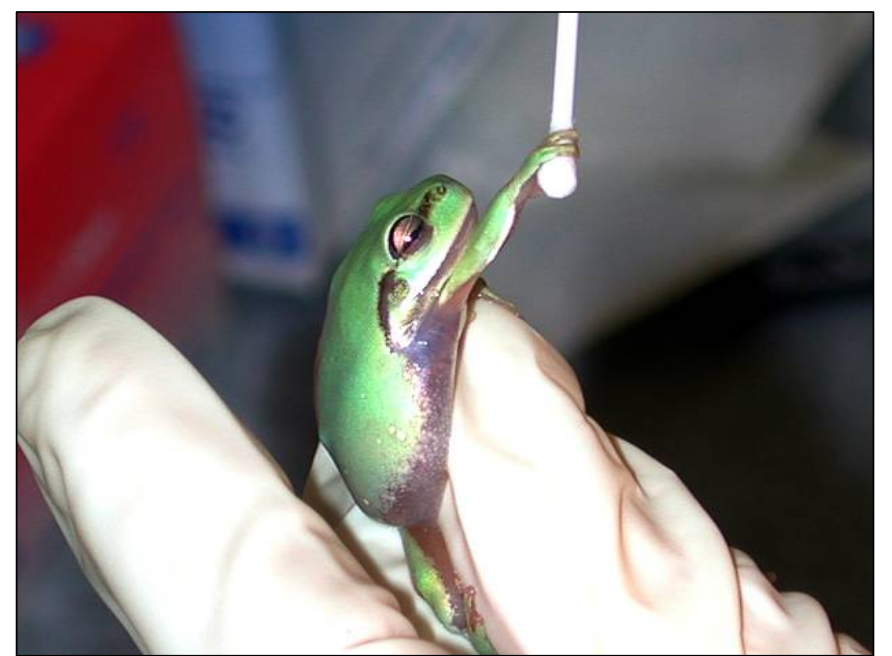

Figure 2. Non destructive sampling of a frog for the presence of $B d$.

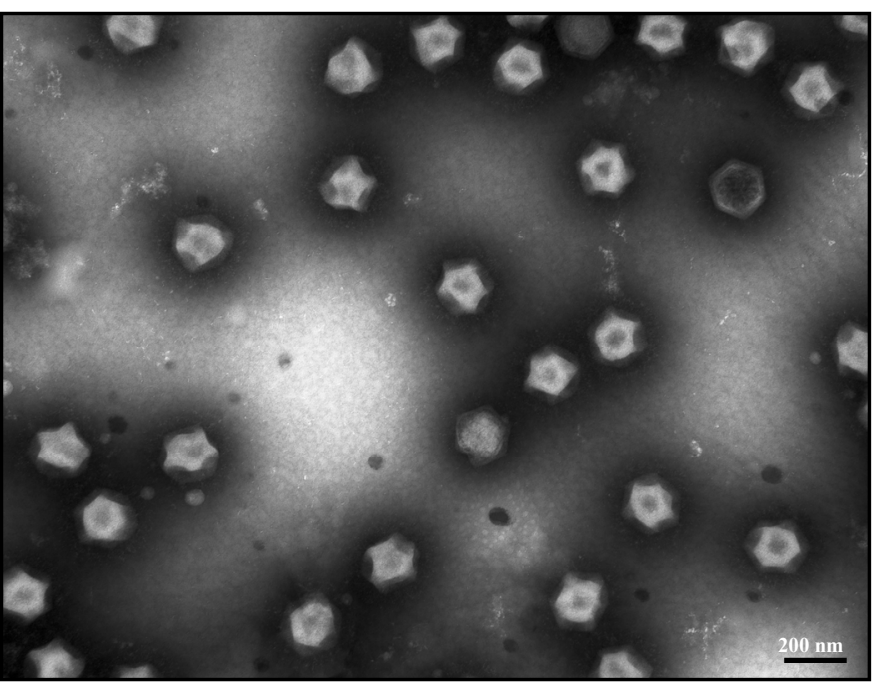

Figure 4. Transmission electron micrograph of the viruses (Fig. 3) prepared for NCEM 